\title{
Distance-related Differences in Critical Times, Protocol Activation and Mortality in a Regional STEMI Network
}

\author{
Balázs Bajka ${ }^{1}$, Marius Orzan ${ }^{1,2}$, Beáta Jakó ${ }^{1}$, István Kovács ${ }^{1,2}$ \\ ${ }^{1}$ Department of Cardiology, County Emergency Clinical Hospital, Tîrgu Mureş, Romania \\ ${ }^{2}$ University of Medicine and Pharmacy, Tîrgu Mures, Romania
}

\section{ARTICLE HISTORY}

Received: 13 March, 2016

Accepted: 11 June, 2016 mortality in STEMI patients presenting to hospitals in the same STEMI network, but located at different distances from the pPCI center. Methods: Four-hundreed sixteen patients with STEMI were studied. Group 1: 101 patients presenting to any of the six regional hospitals in the network located at less than $70 \mathrm{~km}$ from the pPCI center, with a maximum transport time of 30 minutes. Group 2: 81 patients presenting to any of the three territorial hospitals in the network located at 70-150 km from the pPCI center, with a transport time between 30 and 70 minutes. Group 3: 93 patients presenting to any of the four territorial hospitals in the network located at $150-250 \mathrm{~km}$ from the pPCI center, with a transport time between 70 and 150 minutes. Group 4: 141 patients presenting directly to the emergency room of the pPCI center. The following time intervals were recorded: presentation time (PT), from the onset of symptoms to arrival at the pPCI center; protocol initiation time (PIT), from arrival at the PPCI center to STEMI protocol initiation; ischemic time (IT), from the onset of symptoms to repermeabilisation; door to balloon time (DTB), from arrival in the pPCI center to balloon. Results: PT showed no significant difference between the groups $-183.08 \pm 25.2$ minutes vs. $199.1 \pm 32.4$ minutes vs. $166.7 \pm 42.5$ minutes vs. $161.91 \pm$ 36.8 minutes, respectively ( $\mathrm{p}=0.4$ ). PIT was significantly lower in Group 3 (61.66 \pm 15.4 minutes in Group 3 vs. $92 \pm 11.5$ minutes in Group 2 vs. 107.4 \pm 12.5 minutes in Group 1, p = 0.002). DTB time was significantly longer for patients presenting directly to the pPCI center compared to those arriving from Zone 1, 2 or 3 hospitals, $86.96 \pm 11.6$ minutes vs. $52.27 \pm 11.2$ minutes vs. $39.94 \pm 10.3$ minutes vs. $43.9 \pm 5.3$ minutes, $\mathrm{p}<0.001)$. Despite the differences in distance to the pPCI center, there was no significant difference in total IT between the groups (Group 1, $344.6 \pm$ 53.4 minutes; Group 2, $369.3 \pm 42.6$ minutes; Group 3, 366.65 \pm 36.4 minutes; and 340.2 \pm 26.9 minutes in the pPCIcenter, $\mathrm{p}=0.2$ ), and this was reflected in similar rates of mortality (Group 1, 3.9\%; Group 2, 3.7\%; Group 3, 3.2\%; and 3.5\% in the pPCI center). Conclusion: A well organized STEMI network can shorten protocol initiation and DTB times, achieving similar ischemic times and resulting in similar mortality rates with the centers located closer to the pPCI center. Early activation of the STEMI protocol could lead to superior results even in areas situated at longer distances from the pPCI center.

Keywords: STEMI network, acute myocardial infarction, STEMI mortality

\section{CORRESPONDENCE}

\section{Marius Orzan}

Str. Gheorghe Marinescu nr. 50

540139 Tîrgu Mureș, Romania

Tel: +40265212 111

E-mail: orzan_marius@yahoo.com 


\section{INTRODUCTION}

Reperfusion therapy with primary PCI is the recommended treatment for all patients with ST segment elevation acute myocardial infarction (STEMI) presenting within twelve hours from the onset of symptoms. ${ }^{1}$ Early reperfusion of the infarct-related coronary artery (IRA) has a significant impact on patient outcomes after a STEMI episode. ${ }^{2}$ Several studies have confirmed a strong association between myocardial ischemic time on one hand, and infarct size and mortality, on the other hand. ${ }^{2-5}$

Reperfusion of the infarct-related artery can be achieved preferably by primary percutaneous coronary intervention (pPCI) or, when this is not available, within the recommended timeframe for logistic reasons, by fibrinolysis. In both cases, the time from coronary occlusion to reperfusion is crucial for the patient's progress.

The European Myocardial Infarction Project Group found that the administration of fibrinolytic therapy in STEMI, one hour earlier, saved fifteen more lives days per 1,000 patients treated, at thirty days. ${ }^{3}$

Boersmaet et al. analysed over 50,000 STEMI patients from twenty-two randomized trials, and showed that the administration of a thrombolytic agent in patients presenting within one hour of the onset of symptoms saved sixty-five more lives per 1,000 patients, compared with patients whose treatment was commenced at a longer period from the onset of symptoms. ${ }^{4}$

The best clinical results of reperfusion by pPCI have been observed in patients treated up to 120 minutes from the onset of symptoms. ${ }^{5-10}$ In 1,400 patients with acute myocardial infarction treated with primary angioplasty, followed-up for thirteen years, Brodie et al. found that thirty-day mortality was lowest with early reperfusion ( $4.3 \%$ when reperfusion has been achieved $<$ two hours vs. 9.2\% when reperfusion has been achieved after more than two hours), and was relatively independent of time to reperfusion after two hours (9.0\% for reperfusion at two to four hours, $9.3 \%$ for reperfusion at four to six hours, and $9.5 \%$ for reperfusion at more than six hours). ${ }^{5}$

The time from the onset of symptoms until reperfusion denotes the myocardial ischemic time. This interval has patient-related and logistics-related components. The time from the onset of symptoms to the first medical contact depends on the patient's ability to recognize and realize the seriousness of the problem. After the first medical contact, the logistics of the healthcare system is responsible for any delay in reperfusion therapy. For this reason, STEMI networks were planned and developed in order to reduce critical time intervals, and bring the STEMI patient as soon as possible in the cath lab. These STEMI networks typically consist of one pPCI center and regional hospitals without pPCI facility, which refers all STEMI patients presenting within twelve hours from the onset of symptoms to this central pPCI. Different time intervals have been expressed in these STEMI networks, such as protocol initiation time (from the presentation of the patient with chest pain until the initiation of the STEMI protocol in the emergency room), transport time (from the referral hospital to the pPCI center), or door-to-ballon time (from the presentation of the patient to the first balloon inflation in the coronary artery).

Benedek et al. analysed the changes in STEMI treatment and the associated mortality rates in Central Romania from 2004 to 2011, as well as the role of the regional STEMI network in increasing the rate of pPCI and decreasing STEMI mortality. Their results showed that the implementation of an STEMI network increased the rate of pPCI from $10.88 \%$ in 2004 to $78.64 \%$ in 2011 and decreased the in-hospital mortality of STEMI patients from $20.73 \%$ to $6.35 \% .^{11}$

The present study aimed to investigate the differences in critical network times and the association with in-hospital mortality of STEMI patients presenting at regional hospitals belonging to the same STEMI network, but located at different distances from the pPCI center.

\section{METHODS}

We carried out an analysis of recorded critical network times related to 416 patients with STEMI, treated with primary PCI in the County Emergency Clinical Hospital of Tîrgu Mureș from January 1 ${ }^{\text {st }}, 2013$ to December 31, 2013.

The inclusion criteria were:

- symptoms of chest pain suggestive of acute myocardial infarction lasting $\geq 30$ minutes, with onset $<12$ hours before hospital admission, plus

- electrocardiographic findings of ST-segment elevation $>0.1 \mathrm{mV}$ in $\geq 2$ leads, plus

- positive cardiac troponin I.

The pPCI center was located in the County Emergency Clinical Hospital of Tîrgu Mureș, which provides twentyfour-hour emergency cardiac care, seven days a week. The pPCI center provides services for a region with 1,133,307 inhabitants and has thirteen referral regional hospitals without catheterization facilities.

Whenever a patient presenting with chest pain and a suspicion of acute coronary syndrome arrived at the emergency room, 12-lead electrocardiography was recorded, 


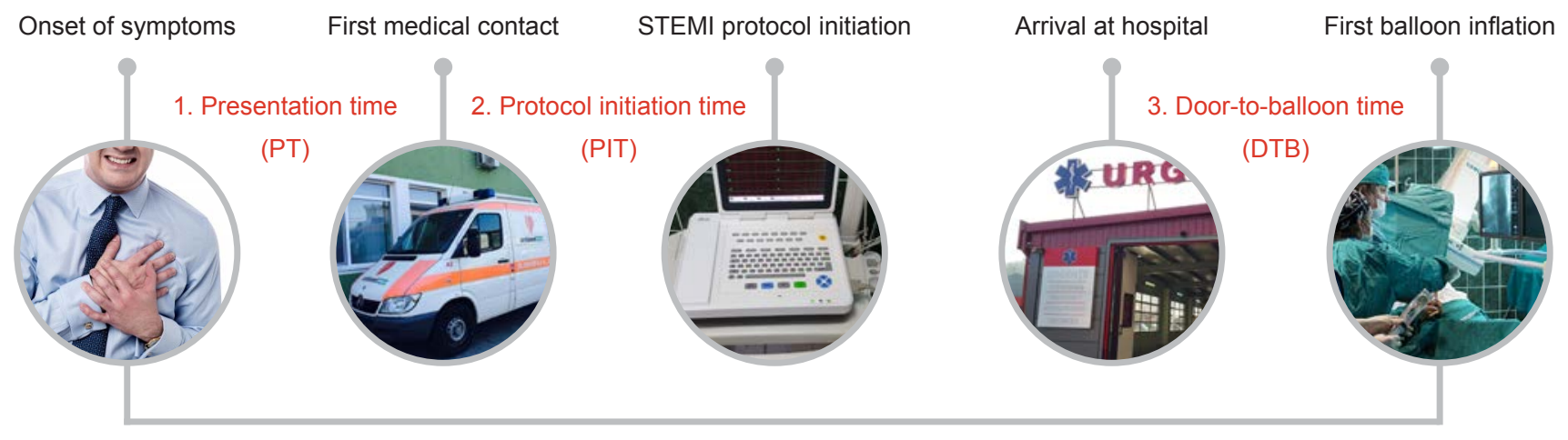

4. Total ischemic time

(IT)

FIGURE 1. Critical time intervals and delays in the network

and cardiac troponin was determined. A positive STEMI diagnosis was followed by the initiation of the STEMI protocol. Activation of the STEMI protocol automatically led to contact with the pPCI center, advising the interventional team of the impending arrival of an STEMI patient. The patient was then transported directly to the catheterization laboratory, thereby bypassing the emergency care unit. The ambulance transfer time varied with the distance from the regional hospital to the pPCI center, between 25 minutes for nearby territorial hospitals to a maximum of 150 minutes for more distant regional hospitals.

The algorithm was different for patients presenting directly at the pPCI center. These patients were first examined in the emergency unit, and whenever an acute coronary syndrome was suspected, a cardiologist was called to confirm the diagnosis. As soon as the STEMI diagnosis was confirmed, the cardiac catheterization laboratory was prepared, the patient was transferred there, and interventional treatment was initiated.

The 416 STEMI patients included in the present study were divided into four groups according to the distance from the regional hospital to the pPCI center.

Group 1: 101 patients presenting to any of the six territorial hospitals in the network located at $<70 \mathrm{~km}$ from the pPCI center (Zone 1), with a maximum transport time of 30 minutes.

Group 2: 81 patients presenting to any of the three territorial hospitals in the network located at $70-150 \mathrm{~km}$ from the pPCI center (Zone 2), with a transport time between 30 and 70 minutes.

Group 3: 93 patients presenting to any of the four territorial hospitals in the network located at $150-250 \mathrm{~km}$ from the pPCI center (Zone 3), with a transport time between 70 and 150 minutes.
Group 4: 141 patients presenting directly to the emergency room of the pPCI center.

The following time intervals were compared (Figure 1):

- presentation time (PT);

- protocol initiation time (PIT);

- door-to-balloon time (DTB);

- ischemic time (IT).

\section{DEFINITIONS}

Presentation time was defined as the time from the onset of symptoms to the first medical contact. The first medical contact (FMC) was defined as the point at which the patient was either initially assessed by medical personnel in the pre-hospital setting, or the patient arrived at the hospital emergency department.

Protocol initiation time was defined as the time from the first medical contact to the initiation of the STEMI protocol.

The door-to-balloon time (DTB) was defined as the time between arrival at the PPCI center and balloon inflation.

Ischemic time (IT) was defined as the time from the onset of symptoms to revascularization.

The study endpoint was the in-hospital mortality of the patients presenting directly to the primary PCI center or referred from the territorial hospitals located at different distances from the pPCI center.

The study protocol was approved by the ethics committee of the institution where the study was conducted, and all patients gave their informed consent for participation in the study. The study was carried out in accordance with the principles stipulated in the Declaration of Helsinki. 


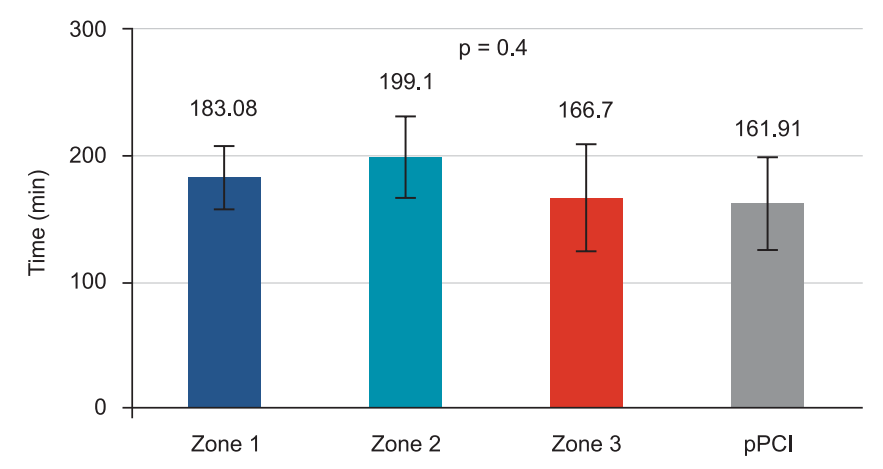

FIGURE 2. Presentation time at the $\mathrm{PPCl}$ center or regional hospitals

\section{STATISTICAL ANALYSIS}

The data was statistically analyzed by using GraphPad Prism 6.0. Quantitative values were expressed as mean and standard deviation. Statistical comparisons between the two study groups were performed using the $\mathrm{Chi}^{2}$ test, and the relative performance of each test was evaluated with the 95\% confidence interval (CI). The level of significance was set at alpha $=0.05$, against which the $\mathrm{p}$ values of each test are compared.

\section{RESULTS}

\section{PRESENTATION TIME}

Presentation time (PT) showed no significant difference between the four groups. This time was higher among patients presenting to the territorial hospital from Zone 2 and Zone 1, compared with patients referred from the territorial hospital located in Zone 3 or those admitted directly to the PPCI center. These differences were not statistically significant, being $183.08 \pm 25.2$ minutes vs. 199.1

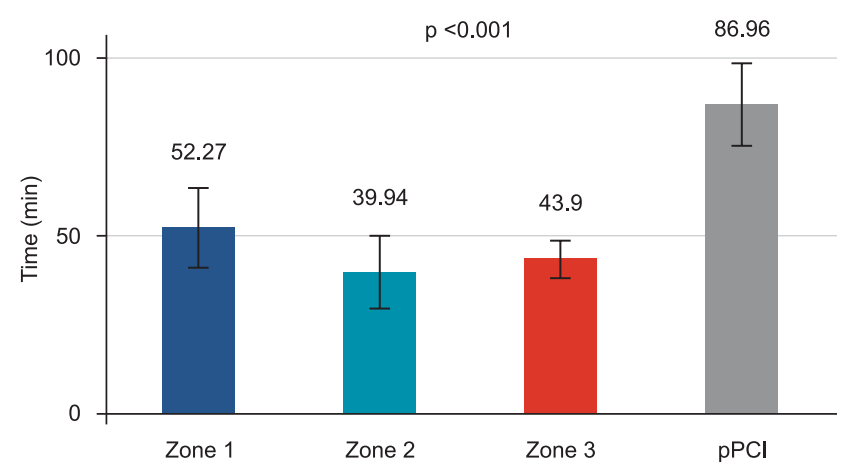

FIGURE 4. Door-to-balloon time of patients presenting at the $\mathrm{PPCl}$ center or regional hospitals

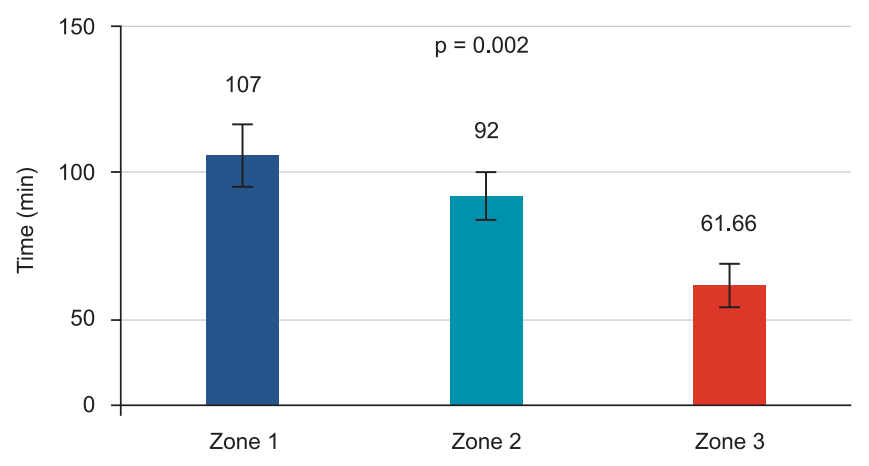

FIGURE 3. Protocol initiation time in the regional hospitals

\pm 32.4 minutes vs. $166.7 \pm 42.5$ minutes vs. $161.91 \pm 36.8$ minutes respectively $(p=0.4)$ (Figure 2 ).

\section{PROTOCOL INITIATION TIME}

Protocol initiation time (PIT) was significantly lower in patients referred from the territorial hospitals located in Zone $3-61.66 \pm 11.2$ minutes in Zone 3 vs. $92 \pm 10.5$ minutes in Zone 2 vs. $107 \pm 13.4$ minutes in Zone $1(p=0.002)$ (Figure 3).

\section{DOOR TO BALLOON TIME}

DTB time was significantly longer for patients presenting directly to the pPCI center compared to those arriving from Zone 1, 2 or 3 hospitals (86.96 \pm 11.6 minutes vs. $52.27 \pm 11.2$ minutes vs. $39.94 \pm 10.3$ minutes vs. $43.9 \pm 5.3$ minutes respectively, $\mathrm{p}<0.001$ ) (Figure 4).

\section{TOTAL MYOCARDIAL ISCHEMIC TIME}

Total ischemic time (IT) was not significantly different

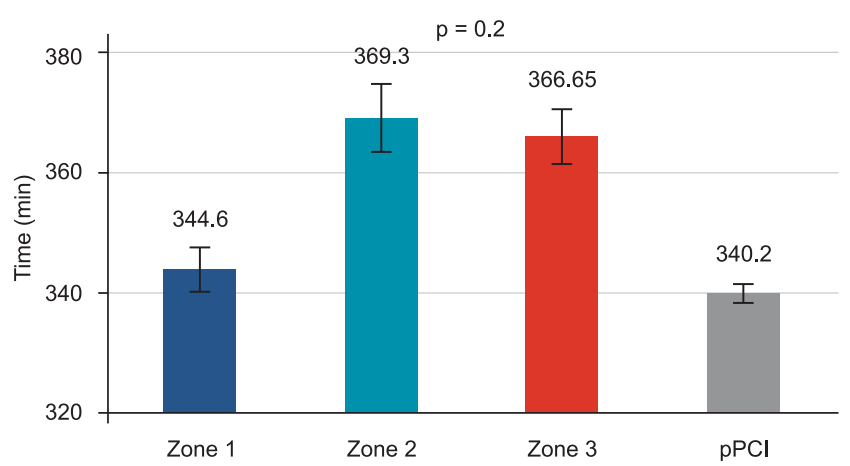

FIGURE 5. Total ischemic time of STEMI patients 


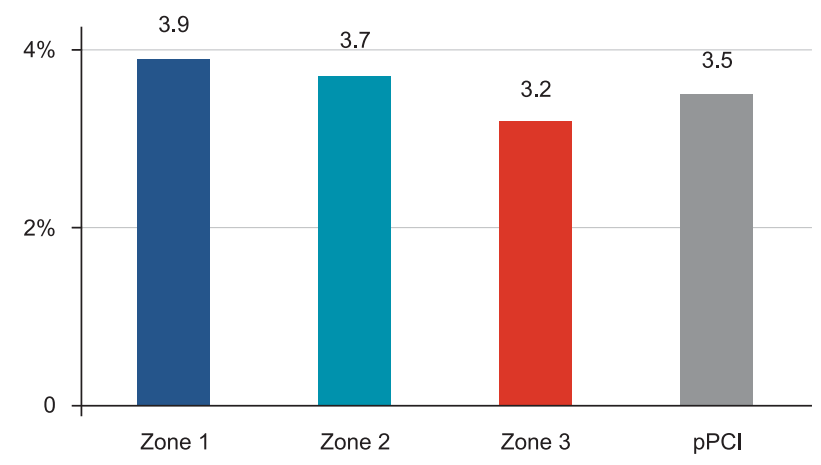

FIGURE 6. Mortality rates of STEMI patients

between the groups - 344.6 \pm 53.4 minutes in Zone 1 , $369.3 \pm 42.6$ minutes in Zone 2, $366.65 \pm 36.4$ minutes in Zone 3 , and $340.2 \pm 26.9$ minutes in the pPCI center $(\mathrm{p}=$ 0.2) (Figure 5).

\section{IN-HOSPITAL MORTALITY OF STEMI PATIENTS}

Mortality rates were similar in all the study groups. The lowest mortality was recorded in STEMI patients presenting to the territorial hospitals located in Zone 3. There were no significant differences between the groups regarding inhospital mortality, this being 3.5\% in the pPCI center, 3.9\% in Zone $1,3.7 \%$ in Zone 2 and 3.2\% in Zone 3 (Figure 6).

\section{DISCUSSIONS}

Myocardial ischemic time is a powerful determinant of mortality in STEMI patients. Therefore, reduction of the total ischemic time should be the primary goal of network organization in an STEMI network.

There are several critical time intervals which need to be considered in reducing the total ischemic time of STEMI patients. The first is the time from the infarctrelated artery occlusion to the onset of symptoms. There is a certain period which elapses from the occlusion of the infarct-related coronary artery to the development of symptoms of myocardial infarction. ${ }^{12}$ The second is the time from the onset of symptoms to the first medical contact. This depends on the ability of the patient to realize the seriousness of the problem and to communicate with an emergency service. The present results showed long presentation times (>160 minutes) in all four groups, with no significant differences between the groups. In a UK study, the median interval from the onset of symptoms to hospital presentation was 2.2 hours. ${ }^{13}$ In the United States, a survey of 18,928 STEMI patients found that $50 \%$ of patients were delayed for more than four hours before they reached a hospital. ${ }^{14}$ These results reflect the overall published results, and indicate a clear need to educate the population in earlier recognition of the signs and symptoms of STEMI, as well as the need for a quick patient transfer to an emergency department whenever there is a suspected acute myocardial infarction.

The protocol initiation time (PIT) in the present study was significantly lower in patients referred from the territorial hospitals located in Zone 3, where the logistic organization of the network proved to be superior to other zones. As this time is a critical component of the systemrelated delay, this resulted in a decreased total ischemic time. At the initiation of the protocol, the immediate administration of aspirin, heparin, and PY12 inhibitors can have a favourable effect on reperfusion. In some circumstances, these drugs can result in early recanalization of the infarct-related artery, which may be present before mechanical reperfusion occurs. ${ }^{15}$

A surprising observation of the present study was the significant difference recorded in door-to-balloon times between the patients presenting directly to the pPCI center and those presenting to the territorial hospitals (87 minutes vs. 44 minutes). This is explained by the fact that those who presented to the territorial hospitals were transported directly to the catheterization laboratory when they arrived in the pPCI center, bypassing the emergency department, as a result of pre-warning of the catheterization laboratory. Patients presenting directly to the pPCI center wasted precious time in the emergency department, where various medical procedures such as blood sampling and record completions were undertaken.

Anderson et al., analysing the data of 33,901 STEMI patients, made a similar observation. They noted that patients transferred directly to a catheterization laboratory had significantly lower DTB times compared to patients transferred first to the emergency department (median 191 minutes vs. 116 minutes), and this was reflected in lower mortality for the patients referred directly to the catheterization laboratory. ${ }^{16,17}$

Another surprising result was the similar ischemic times of the patients presenting to the territorial hospitals and the pPCI center $(p=0.2)$. Despite the longer transportation time from the territorial hospitals located in Zone 3 to the pPCI center, patients presenting to the referral hospitals from this area had a similar total ischemic time to those who presented to the pPCI center. This results from significantly lower protocol initiation times and door-toballoon times recorded in the well-functioning network in this area. This observation indicates a clear need for a 
better logistic organization in the pPCI center to reduce the DTB and PIT time intervals for patients who present directly to the pPCI center.

De Luca ${ }^{\text {et al }}$. analysed the relationship between myocardial ischemic time and one-year mortality in 1,800 STEMI patients treated with $\mathrm{pPCI}$. They found that the relative mortality risk increased by $7.5 \%$ for every thirty-minute delay before reperfusion..$^{18}$ As a result of the similar total ischemic times, the STEMI patients referred from the territorial hospitals to the pPCI center had similar mortality rates (3.9 vs. 3.7 vs 3.2 vs 3.5 ), with a slightly lower mortality in cases of the patients presenting directly to the pPCI center. This shows that reducing the system delay by a well-organized STEMI network can provide a similar chance of survival for patients living at a distance from the pPCI center compared to patients who present directly to the pPCI center.

In a retrospective study, Swaminathan et al. analysed 82,678 STEMI patients who underwent primary PCI and were enrolled in the National Cardiovascular Data Registry, and found that in-hospital mortality for patients with significant network-related delays was significantly higher than for patients who did not experience any significant network-related delay ( $15.1 \%$ vs. $2.5 \%$ ). ${ }^{19}$

A system-related delay has been proved to be linked with long-term mortality. Terkelsen et al. in a follow-up study on 6,209 patients, suggested that a system delay was independently associated with mortality, as were protocol initiation time, transport time and door-to-balloon delay. A system-related delay of fewer than sixty minutes corresponded to a long-term mortality rate of $15.4 \%$ compared to a rate of $30.8 \%$ when the delay was 181 to 360 minutes. ${ }^{20,21}$

In agreement with the observations of De Luca et al., the present study confirmed that total myocardial ischemic time is the most relevant time interval influencing in-hospital mortality in STEMI. ${ }^{18}$ Total ischemic time has a greater impact than PT, PIT, transport time or DTB. Despite the differences recorded in all these components, the in-hospital mortality did not show any significant differences between the groups, as the total ischemic time was approximately the same in all groups. The results also emphasise the importance in reducing the true ischemic time by decreasing the whole system delay and its components, instead of focusing only on the door-to-balloon delay, as a performance measure for triaging patients for primary percutaneous coronary intervention.

\section{CONCLUSIONS}

A well-organized STEMI network can shorten protocol initiation and DTB times, thus reaching similar ischemic times and presenting similar mortality rates with the centers located closer to the pPCI center. Early activation of the STEMI protocol could lead to superior results even in areas situated at longer distances from a pPCI center.

\section{CONFLICT OF INTEREST}

Nothing to declare.

\section{ACKNOWLEDGEMENT}

This paper was published under the frame of European Social Found, Human Resources Development Operational Programme 2007-2013, project no. POSDRU/159/1.5/S/133377.

\section{REFERENCES}

1. Task Force on the management of ST-segment elevation acute myocardial infarction of the European Society of Cardiology (ESC), Steg PG, James SK, et al. ESC Guidelines for the management of acute myocardial infarction in patients presenting with ST-segment elevation. Eur Heart J. 2012;33:2569-2619. doi: 10.1093/eurheartj/ehs215.

2. Weaver WD. Time to thrombolytic treatment: factors affecting delay and their influence on outcome. J Am Coll Cardiol. 1995;25:3S-9S.

3. The European Myocardial Infarction Project Group. Prehospital thrombolytic therapy in patients with suspected acute myocardial infarction. N Engl J Med. 1993;329:383-389.

4. Boersma E, Maas AC, Deckers JW, et al. Early thrombolytic treatment in acute myocardial infarction: reappraisal of the golden hour. Lancet. 1996;348:771-775. doi: 10.1016/S01406736(96)02514-7.

5. Brodie BR, Stuckey TD, Wall TC, et al. Importance of time to reperfusion for 30-day and late survival and recovery of left ventricular function after primary angioplasty for acute myocardial infarction. J Am Coll Cardiol. 1998;32:1312-1319.

6. Brodie BR, Hansen C, Stuckey TD, et al. Door-to-balloon time with primary percutaneous coronary intervention for acute myocardial infarction impacts late cardiac mortality in highrisk patients and patients presenting early after the onset of symptoms. J Am Coll Cardiol. 2006;47:289-295.

7. Francone M, Bucciarelli-Ducci C, Carbone I, et al. Impact of primary coronary angioplasty delay on myocardial salvage, infarct size, and microvascular damage in patients with ST-segment elevation myocardial infarction: insight from cardiovascular magnetic resonance. J Am Coll Cardiol. 2009;54:2145-2153. doi: 10.1016/j.jacc.2009.08.024.

8. Tarantini G, Cacciavillani L, Corbetti F, et al. Duration of ischemia is a major determinant of transmurality and severe microvascular obstruction after primary angioplasty: a study performed with contrast-enhanced magnetic resonance. J Am Coll Cardiol. 2005;46:1229-1235. doi: 10.1016/j. jacc.2005.06.054.

9. Postma S, Dambrink J, Marcel AT, et al. The influence of system delay on 30-day and on long-term mortality in 
patients with anterior versus non-anterior ST-segment elevation myocardial infarction: a cohort study. Open Heart 2015;2. doi:10.1136/openhrt-2014-000201.

10. Brodie BR, Webb J, Stone GW, et al. Impact of time to treatment on myocardial reperfusion and infarct size with primary percutaneous coronary intervention for acute myocardial infarction (from the EMERALD Trial). Am J Cardiol. 2007;99:1680-1686. doi: 10.1016/j.amjcard.2007.01.047.

11. Benedek I, Gyongyosi M, Benedek T. A prospective regional registry of ST-elevation myocardial infarction in Central Romania: impact of the Stent for Life Initiative recommendations on patient outcomes. Am Heart J. 2013;166:457-465. doi: 10.1016/j.ahj.2013.03.033.

12. Fischell TA, Fischell DR, Fischell RE, et al. Potential of an intracardiac electrogram for the rapid detection of coronary artery occlusion. Cardiovasc Revasc Med. 2005;6:14-20. doi: 10.1016/j.carrev.2005.05.002.

13. Horne R, James D, Petrie K, et al. Patients' interpretation of symptoms as a cause of delay in reaching hospital during acute myocardial infarction. Heart. 2000;83:388-393.

14. Tra J, van der Wulp I, de Bruijne MC, Wagner C. Exploring the treatment delay in the care of patients with ST-elevation myocardial infarction undergoing acute percutaneous coronary intervention: a cross-sectional study. BMC Health Services Research. 2015;15:340. doi:10.1186/s12913-015-0993-y.

15. Vlaar PJ, Svilaas T, Damman K, et al. Impact of pretreatment with clopidogrel on initial patency and outcome in patients treated with primary percutaneous coronary intervention for ST-segment elevation myocardial infarction: a systematic review. Circulation. 2008;118:1828-1836. doi: 10.1161/ CIRCULATIONAHA.107.749531.
16. Anderson LL, French WJ, Peng SA, et al. Direct Transfer From the Referring Hospitals to the Catheterization Laboratory to Minimize Reperfusion Delays for Primary Percutaneous Coronary Intervention: Insights From the National Cardiovascular Data Registry. Circ Cardiovasc Interv. 2015;8:e002477. doi: 10.1161/ CIRCINTERVENTIONS.114.002477.

17. Kutcher MA. Door-to-Balloon Time as a Process Metric for Treatment of ST-Segment Elevation Myocardial Infarction Time to "Tap Out". JACC Cardiovasc Interv. 2015;8:1975-1977. doi: 10.1016/j.jcin.2015.10.007.

18. De Luca G, Suryapranata H, Ottervanger JP, et al. Time delay to treatment and mortality in primary angioplasty for acute myocardial infarction: every minute of delay counts. Circulation. 2004;109:1223-1225. doi: 10.1161/01. CIR.0000121424.76486.20.

19. Swaminathan R, Wang T, Kaltenbach L, et al. Nonsystem Reasons for Delay in Door-to-Balloon Time and Associated In-Hospital Mortality A Report From the National Cardiovascular Data Registry. J Am Coll Cardiol. 2013;61:16881695. doi:10.1016/j.jacc.2012.11.073.

20. Terkelsen CJ, Sorensen JT, Maeng M, et al. System delay and mortality among patients with STEMI treated with primary percutaneous coronary intervention. JAMA. 2010;304:763-771. doi: 10.1001/jama.2010.1139.

21. Terkelsen CJ, Lassen JF, Nørgaard BL, et al. Reduction of treatment delay in patients with ST-elevation myocardial infarction: impact of pre-hospital diagnosis and direct referral to primary percutanous coronary intervention. Eur Heart J. 2005;26:770-777. doi: 10.1093/eurheartj/ehi100. 